\title{
Can dorzolamide/timolol-fixed combination effectively treat primary open-angle glaucoma? A protocol for systematic review and meta-analysis
}

\section{Yan-Xiu Qi}

First Affiliated Hospital of Jiamusi University

Hong-wei Liu ( $\square$ hongwei200411@163.com )

First Affiliated Hospital of Jiamusi University

\section{Xing-jie Su}

First Affiliated Hospital of Jiamusi University

\section{Lin Han}

The 163th Hospital of the People's Liberation Army Joint Service Support Force

\section{Protocol}

Keywords: Primary open-angle glaucoma, dorzolamide, timolol, efficacy, safety

Posted Date: May 11th, 2020

DOI: https://doi.org/10.21203/rs.3.rs-27271/v1

License: (a) (i) This work is licensed under a Creative Commons Attribution 4.0 International License.

Read Full License

Version of Record: A version of this preprint was published at Medicine on November 20th, 2020. See the published version at https://doi.org/10.1097/MD.0000000000023245. 


\section{Abstract}

Background: Primary open-angle glaucoma (POAG) is a very common disorder, and it is the second leading reason that leads to blindness worldwide after cataracts. Previous studies have reported that dorzolamide/timolol-fixed combination (DTFC) can be used to treat patients with POAG. However, there are still inconsistent results. Thus, this study will systematically investigate the efficacy and safety of DTFC for the treatment of patients with POAG.

Methods: A comprehensive search will be carried out in the electronic databases of Cochrane Library, MEDLINE, EMBASE, CINAHI, ACMD, China National Knowledge Infrastructure, and Wanfang Data from origin to the March 1, 2020. There are no limitations related to the language and publication status. Only randomized controlled trials that assessed the efficacy and safety of DTFC for the treatment of POAG will be included. Two researchers will independently undertake record selection, data extraction, and methodological quality assessment. Any divisions will be solved by discussion with a third researcher. We will perform statistical analysis using RevMan 5.3 software

Discussion: This study will summarize present evidence to identify the efficacy and safety of DTFC for the treatment of POAG through mean intraocular pressure, best corrected visual acuity, contrast sensitivity, bioelectric activity of the retina, rate of progression of glaucoma, quality of life, and adverse events. The results of this study will help to determine whether DTFC is effective and safety for the treatment of POAG.

Systematic review registration: INPLASY202040120.

\section{Background}

Primary open-angle glaucoma (POAG) is the most types of glaucoma [1-4]. It is associated with elevated intraocular pressure (IOP) and damage to the optic nerve [4-5], which finally results in irreversible vision loss [6-9]. Conventional managements mainly focus on lowering IOP [10-12]. Among them, dorzolamide/timolol-fixed combination (DTFC) is available for the treatment of patients with POAG [1321]. However, insufficient literature data regarding DTFC for the treatment of POAG has been supplied. Thus, this study will systematically assess the efficacy and safety of DTFC for the management of POAG.

\section{Methods And Analysis}

\section{Study registration}

This study was registered on INPLASY202040120. It has been reported according to the guidelines of Preferred Reporting Items for Systematic Reviews and Meta-analysis Protocol [22].

\section{Study selection criteria}




\section{Type of studies}

Only randomized controlled trials (RCTs) will be included in this study, which explored the efficacy and safety of DTFC for the treatment of POAG. We will exclude any other studies, such as animal study, review, case report, uncontrolled trial, non-RCTs and quasi-RCTs.

\section{Type of participants}

Any patients who were diagnosed as POAG in spite of country, race, gender, age, and severity of POAG will be included.

\section{Type of interventions}

In the experimental group, all patients who received DTFC as their solely treatment will be included. In the control group, subjects could receive any treatments without restrictions. However, we will not consider study which involved any forms of DTFC as its comparator.

\section{Types of outcomes}

\section{Primary outcomes}

Mean intraocular pressure; and

Best corrected visual acuity.

\section{Secondary outcomes}

Contrast sensitivity;

Bioelectric activity of the retina;

Rate of progression of glaucoma;

Quality of life (as assessed by 36-Item Short Form Survey); and

Adverse events.

\section{Data sources and search strategy}

A comprehensive search will be performed from origin to the March 1, 2020 in the electronic databases of Cochrane Library, MEDLINE, EMBASE, CINAHI, ACMD, China National Knowledge Infrastructure, and Wanfang Data. We will not apply limitations related to the language and publication status. We will only consider RCTs that appraised the efficacy and safety of DTFC for the treatment of POAG. We have summarized search strategy sample for Cochrane Library (table 1), and will create similar search 
strategies for other electronic databases. In addition, we will also scrutinize other sources, such as Google Scholar, conference proceedings, and reference lists of included trials.

\section{Study selection}

Two researchers will independently identify titles/abstracts of sought citations to remove unrelated studies. Then, full papers of potential trials will be further inspected against all eligibility criteria. If any differences are identified, we will invite a third researcher to solve them by discussion. All reasons for excluded studies will be listed. We will exert the process of study selection in a flowchart.

\section{Data extraction and management}

Two researchers will independently extract data from all included RCTs using a predefined standard data extraction form. Any divergences between two of them will be resolved through discussion with a third researcher. The extracted information consists of study information (e.g. title, first author), patient characteristics (e.g. diagnosis criteria, eligibility criteria), study methods (e.g. sample size, randomization); details of intervention and controls (e.g. treatment types, dosage), outcome measurements, adverse events, and conflict of interest.

\section{Dealing with missing data}

We will contact primary authors to request any unclear or missing data. If it can not be obtained, we will analyze available data using intention-to-treat analysis.

\section{Risk of bias assessment}

Two researchers will independently appraise methodological quality for all eligible RCTs using Cochrane Handbook for Systematic Reviews of Interventions Tool [23]. Any disagreements will be resolved by a third researcher by consultation and a consensus will be reached.

\section{Quality of evidence rating}

Two researchers will independently assess the overall strength of the evidence using Grading of Recommendations Assessment, Development and Evaluation tool [24]. Its results will be demonstrated in the table of Summary of Findings. A third researcher will help to solve any disagreements.

\section{Statistical analysis}

RevMan 5.3 software (Cochrane, London, UK) will be utilized to perform statistical analysis. We will estimate continuous outcome values using mean difference or standardized mean difference with $95 \%$ confidence intervals (Cls), and dichotomous outcome values using risk ratio with $95 \% \mathrm{Cls}$. We will examine heterogeneity using $I^{2}$ statistic. We will use a fixed-effect model to pool the data $\left(I^{2} \leq 50 \%\right)$, and will utilize a random-effect model $\left(I^{2}>50 \%\right)$ to synthesize the data. If $I^{2} \leq 50 \%$ and sufficient number of 
eligible study is included, we will carry out meta-analysis. Otherwise, if $I^{2}>50 \%$, we will conduct a subgroup analysis to investigate the sources of heterogeneity.

\section{Additional analysis}

A subgroup analysis will be carried out according to the variations in study and patient characteristics, different types of interventions and controls, and different study quality.

A sensitivity analysis will be conducted to test the stability of conclusions by eliminating low quality trials.

If necessary, we will perform a funnel plot and Egger's regression test to check reporting bias when over 10 RCTs are included.

\section{Dissemination}

This study will be published in print, conferences or by peer-reviewed journals.

\section{Amendments}

Any changes to this protocol will be noted with reference to saved searches and analysis.

\section{Discussion}

POAG is the most common of glaucoma, which often results in vision loss [1-4]. The management approach of DTFC becomes very important in treating POAG. However, as far as we know, rarely data at systematic level is available on the efficacy and safety of DTFC for the treatment of POAG. Thus, it is important to establish the use of DTFC modality in the management of patients with POAG. We believe that the findings of this study may provide evidence to determine whether DTFC is effective and safe for the treatment of POAG. Its results may benefit patients, clinicians, and health-related decision-makers.

\section{Abbreviations}

POAG, primary open-angle glaucoma; DTFC, dorzolamide/timolol-fixed combination; RCTs, randomized controlled trials; Cls, confidence intervals.

\section{Declarations}

Ethics approval and consent to participate: Not applicable

Consent for publication: Not applicable

Availability of data and material: Data sharing is not applicable to this article as no datasets were generated or analyzed during the current protocol 
Competing interests: All authors declare that they have no competing interests.

Funding: This work was supported in part by the Scientific Research Project of Heilongjiang Provincial Department of Health (2013257), Jiamusi University Project (13Z1201547), and Scientific Research Projects of Heilongjiang Provincial Health and Health Committee (2019-327). The funders did not take part in the design, execution, or writing of the study.

Authors' contributions: YXQ, HWL, XJS and LH conceived the study. XJS and LH contributed with the clinical background and expertise. YXQ, HWL, and XJS contributed with the analytical plan and the bias assessment approach. YXQ and HWL performed the literature search plan. YXQ, HWL, XJS and LH drafted the protocol. All authors revised the protocol and approved the final version. DS supervised the study

Acknowledgements: Not applicable

Availability of data and materials: Data sharing is not applicable to this article as no datasets were generated or analyzed during the current protocol

Competing interests: All authors declare that they have no competing interests.

\section{References}

1. Erichev VP, Egorov EA. On pathogenesis of primary open-angle glaucoma. Vestn Oftalmol 2014; 130(6):98-104.

2. Weinreb RN, Leung CK, Crowston JG, et al. Primary open-angle glaucoma. Nat Rev Dis Primers 2016; 2:16067.

3. Hoffmann EM, Prokosch-Willing V. Primary Open Angle Glaucoma. Klin Monbl Augenheilkd 2017; 234(11):1407-22.

4. Yang Y, Ma QY, Yang Y, et al. Evidence-based practice guideline of Chinese herbal medicine for primary open-angle glaucoma (qingfeng -neizhang). Medicine (Baltimore) 2018; 97(13):e0126.

5. Kazarian EE. Current methods for monitoring primary open-angle glaucoma. Vestn Oftalmol 2009; 125(4):40-4.

6. Sharts-Hopko NC, Glynn-Milley C. Primary open-angle glaucoma. Am J Nurs 2009; 109(2):40-7.

7. Kwon YH, Fingert JH, Kuehn MH, Alward WL. Primary open-angle glaucoma. N Engl J Med 2009; 360(11):1113-24.

8. . Hazin R, Hendrick AM, Kahook MY. Primary open-angle glaucoma: diagnostic approaches and managementJ Natl Med Assoc 2009; 101(1):46-50.

9. Gemenetzi M, Yang Y, Lotery AJ. Current concepts on primary open-angle glaucoma genetics: a contribution to disease pathophysiology and future treatment. Eye (Lond) 2012; 26(3):355-69. 
10. Choquet $\mathrm{H}$, Wiggs JL, Khawaja AP. Clinical implications of recent advances in primary open-angle glaucoma genetics. Eye (Lond) 2020; 34(1):29-39.

11. Liebmann JM, Lee JK. Current therapeutic options and treatments in development for the management of primary open-angle glaucoma. Am J Manag Care 2017; 23(15 Suppl):S279-92.

12. Rait JL. Management of ocular hypertension and primary open angle glaucoma. Clin Exp Optom 2000; 83(3):136-44.

13. Rolle T, Tofani F, Brogliatti B, Grignolo FM. The effects of dorzolamide $2 \%$ and dorzolamide/timolol fixed combination on retinal and optic nerve head blood flow in primary open-angle glaucoma patients. Eye (Lond) 2008; 22(9):1172-9.

14. Cvenkel B, Stewart JA, Nelson LA, Stewart WC. Dorzolamide/timolol fixed combination versus latanoprost/timolol fixed combination in patients with primary open-angle glaucoma or ocular hypertension. Curr Eye Res 2008; 33(2):163-8.

15. Konstas AG, Quaranta L, Yan DB, et al. Twenty-four hour efficacy with the dorzolamide/timolol-fixed combination compared with the brimonidine/timolol-fixed combination in primary open-angle glaucoma. Eye (Lond) 2012; 26(1):80-7.

16. Kozobolis V, Panos GD, Konstantinidis A, Labiris G. Comparison of dorzolamide/timolol vs brinzolamide/brimonidine fixed combination therapy in the management of primary open-angle glaucoma. Eur J Ophthalmol 2017; 27(2):160-3.

17. Babić N, Andreić V, Miljković A, Grković D, Jovanović P. Comparison of the efficacy and safety of fixed combination travoprost/timolol and dorzolamide/ timolol in patients with primary open-angle glaucoma and ocular hypertension. Srp Arh Celok Lek 2013; 141(7-8):441-6.

18. Eren MH, Gungel H, Altan C, Pasaoglu IB, Sabanci S. Comparison of dorzolamide/timolol and latanoprost/timolol fixed combinations on diurnal intraocular pressure control in primary open-angle glaucoma. J Ocul Pharmacol Ther 2012; 28(4):381-6.

19. Gulkilik G, Oba E, Odabası M. Comparison of fixed combinations of dorzolamide/timolol and brimonidine/timolol in patients with primary open-angle glaucoma. Int Ophthalmol 2011;31(6):44751.

20. Pajic B, Pajic-Eggspuehler B, Häfliger IO. Comparison of the effects of dorzolamide/timolol and latanoprost/timolol fixed combinations upon intraocular pressure and progression of visual field damage in primary open-angle glaucoma. Curr Med Res Opin 2010; 26(9):2213-9.

21. Janulevicienë I, Harris A, Kagemann L, Siesky B, McCranor L. A comparison of the effects of dorzolamide/timolol fixed combination versus latanoprost on intraocular pressure and pulsatile ocular blood flow in primary open-angle glaucoma patients. Acta Ophthalmol Scand 2004; 82(6):730-7.

22. Shamseer L, Moher D, Clarke M, et al. Preferred reporting items for systematic review and metaanalysis protocols (PRISMA-P) 2015: elaboration and explanation. BMJ 2015; 349:g7647.

23. Higgins JP, Altman DG, Gøtzsche PC, et al. The Cochrane Collaboration's tool for assessing risk of bias in randomised trials. BMJ 2011;343:1-9. 
24. Guyatt GH, Oxman AD, Vist GE, et al. GRADE: an emerging consensus on rating quality of evidence and strength of recommendations. BMJ 2008; 336:924-6.

\section{Table}

Table 1 Search strategy used in Cochrane Library database

\begin{tabular}{|c|c|}
\hline Number & Search terms \\
\hline 1 & Mesh descriptor: (glaucoma, open-angle) explode all trees \\
\hline 2 & 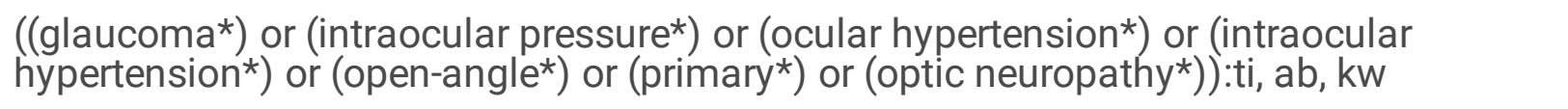 \\
\hline 3 & Or $1-2$ \\
\hline 4 & Mesh descriptor: (timolol) explode all trees \\
\hline 5 & (dorzolamide) explode all trees \\
\hline 6 & 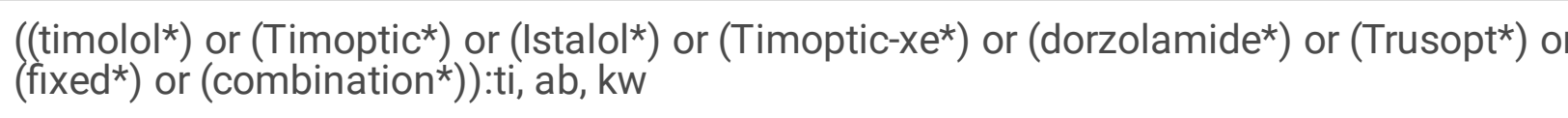 \\
\hline 7 & Or 4-6 \\
\hline 8 & MeSH descriptor: (randomized controlled trials) explode all trees \\
\hline 9 &  \\
\hline
\end{tabular}

Or 8-9 